\title{
Effect of Training in Upper Endoscopic Biopsy
}

\author{
Chang Seok Bang, Gwang Ho Baik*, Jong Hyeok Kim*, Jin Bong Kim, Ki Tae Suk, Jai Hoon Yoon, Yeon Soo Kim, Dong Joon Kim \\ Department of Internal Medicine, Hallym University College of Medicine, Chuncheon, Korea
}

\begin{abstract}
Background/Aims: The optimal number of biopsy samples and the detection rate of neoplastic lesions are not established. This study aimed to assess the current practice patterns and effects of training in upper endoscopic biopsy.

Materials and Methods: From May through July 2013, all the biopsy-proven lesions detected during diagnostic esophagogastroduodenoscopy were enrolled in a single teaching hospital of Korea. Endoscopic findings, including number of biopsied samples, discrepancy rates between endoscopic and histologic diagnosis, and endoscopists' experience, were retrospectively assessed. Results: A total of 1,208 biopsy-proven lesions were enrolled. 76 (6.3\%) lesions were determined to be neoplastic. The neoplasm detection rate of trainees was lower than that of faculty doctors $(4.6 \% v s .7 .7 \%$; OR, $0.57 ; P=0.024)$. The number of biopsied samples was not different between trainees and faculty doctors, although faculty doctors tended to identify more neoplastic lesions. The concordance rates between endoscopic and histologic diagnosis were improved with training for both total and benign lesions, but there was no changes in concordance for neoplastic lesions.
\end{abstract}

Conclusions: Training should be focused on detection of neoplastic lesions so as not to overlook these diseases. (Korean J Helicobacter Up Gastrointest Res 2015;15:33-38)

Key Words: Biopsy; Endoscopy; Neoplasms

\section{INTRODUCTION}

Endoscopic examination is indicated in screening for and diagnosing a variety of gastrointestinal diseases, including neoplastic lesions. In Korea, where stomach cancer is prevalent, high-quality endoscopic examination is necessary to detect pathologic gastrointestinal lesions. Endoscopic biopsy is the gold standard for the confirmation of endoscopic diagnosis, ${ }^{1,2}$ which is usually determined based on the abnormal morphology of the lesions or color changes in the mucosa. ${ }^{3}$ Diagnostic accuracy is known to be improved by training and the use of optical techniques or chromoendoscopy. ${ }^{4}$ The development of endoscopic imaging technologies such as narrow band imaging (NBI), confocal imaging or magnifying techniques can enhance diagnostic accuracy., 3.5

However, inspection via conventional white light endoscopy remains the most routine form of screening endoscopy. Thus, meticulous inspection of tissues from

Received: September 17, 2014 Accepted: November 11, 2014

Corresponding author: Gwang Ho Baik

Department of Internal Medicine, Chuncheon Sacred Heart Hospital, Hallym University College of Medicine, 77 Sakju-ro, Chuncheon 200-704, Korea

Tel: +82-33-240-5821, Fax: +82-33-241-8064, E-mail: baikgh@hallym.or.kt

*These authors contributed equally to this work as corresponding authors. accurate and targeted biopsies is a key factor in diagnosing pathologic lesions. However, the recommended number of biopsy specimens and the quality indices such as detection rate of neoplastic lesions have not been well established. This study aimed to evaluate the current practice pattern and effect of training in conventional upper endoscopic biopsy.

\section{MATERIALS AND METHODS}

\section{Patients}

From May through July 2013, all the biopsy specimens from diagnostic esophagogastroduodenoscopy (EGD) performed in a single teaching hospital of Korea were collected and analyzed. EGDs were performed either as part of routine check-ups or as diagnostic examinations of symptomatic patients. Transferred patients from other hospitals for the further investigation or patients who have biopsy proven lesions were excluded from this study. Specimens from therapeutic procedures, such as endoscopic mucosal resection (EMR) and endoscopic submucosal dissection (ESD), were excluded from this study. Only pinch biopsy specimens were included; specimens from hot biopsies and biopsies by snare or knife were

Copyright $\odot 2015$ Korean College of Helicobacter and Upper Gastrointestinal Research

() The Korean Journal of Helicobacter and Upper Gastrointestinal Research is an Open-Access Journal. All articles are distributed under the terms of the Creative Commons Attribution Non-Commercial License (http://creativecommons.org/licenses/by-nc/3.0) which permits unrestricted non-commercial use, distribution, and reproduction in any medium, provided the original work is properly cited. 
excluded. Biopsies for the detection of Helicobacter pylori were also excluded in this study (Fig. 1).

Endoscopic findings, including location of the lesion, number of biopsy specimens, and endoscopists' experience, were recorded. The endoscopic diagnosis in the procedure report and histologic diagnosis in the pathology report were compared to determine concordance or discrepancy.

Six experienced ( $>6,000$ cases) endoscopist faculty doctors, and three endoscopy fellow in training $(<4$ months of endoscopic training) performed the diagnostic endoscopies. The study protocol was approved by the institutional review board of Chuncheon Sacred Heart Hospital (2014-15).

\section{Endoscopic biopsy and training}

The need for endoscopic biopsy was determined by the endoscopists during the examination, and nurses assisted with the biopsy procedure. Sterilized reusable biopsy forceps were used and were cleaned and sterilized after each procedure. Biopsy specimens were placed in formalin immediately after the procedure and sent to the pathology department for histologic confirmation.

The endoscopic trainees had less than 4 months of experience. The training plan and educational objectives were based on the Korean Association of Internal Medicine guidelines. After observing endoscopies and acquiring basic skills, the trainees performed diagnostic EGDs during the study. The training plan included education of the use of NBI and magnifying systems, and trainees were al- lowed to apply these techniques at will during the examination. The need for endoscopic biopsy and the number of biopsy samples were decided by trainees.

Endoscopic examination was performed by Olympus H260 (Olympus Optical Co., Ltd., Tokyo, Japan). Biopsy was performed by Captura disposable biopsy forcep (DBF-2.4SN-230SP-S; Wilson-Cook Medical Inc., WinstonSalem, NC, USA).

\section{Sample size calculation}

The sample size was calculated as follows: 1 . The detection rate for neoplastic lesions was assumed to be $7 \%$ among faculty doctors and 5\% among trainees based on a preliminary analysis the May 2013 biopsy results. 2. It was determined that 1,031 biopsy lesions would be required to detect differences between faculty doctors and trainees at $5 \%$ probability and a power of $80 \%$. In total, 1,208 lesions satisfying inclusion and exclusion criteria were biopsied between May and July.

\section{Statistical analysis}

Continuous variables were expressed as the median and interquartile range (IQR) because they were not normally distributed; categorical variables were expressed as numbers and percentages. Mann-Whitney and Fisher's exact tests were used to compare two variables, and ANOVA was used to compare three continuous variables. A multivariable logistic regression analysis was performed to assess independent risk factors associated with detection of neoplastic lesion during endoscopy. A $P$ value

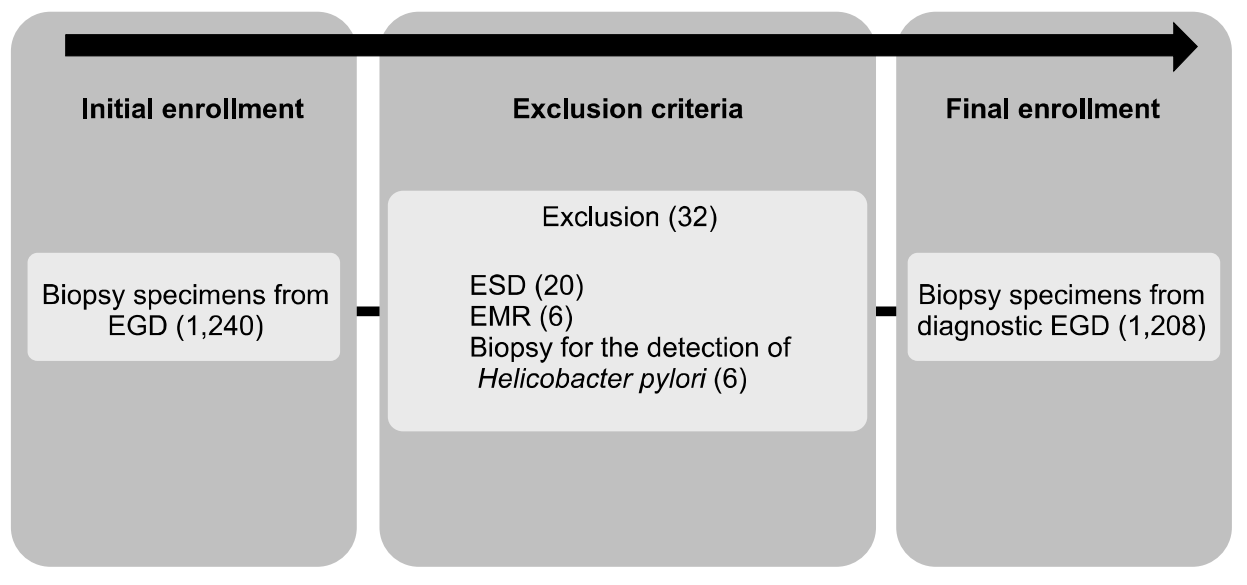

Fig. 1. Flow chart of study design. EGD, esophagogastroduodenoscopy; ESD, endoscopic submucosal dissection; EMR, endoscopic mucosal resection. 
$<0.05$ was adopted as the threshold of statistical significance for all tests. The analyses were performed using PASW Statistics version 18.0 (IBM Co., Armonk, NY, USA).

\section{RESULTS}

\section{Characteristic of biopsied lesions}

During the study period, a total of 1,208 lesions were biopsied. The clinical characteristics of these lesions are shown in Table 1. The median age of patients was as follows: 60 years overall, 59 years in patients biopsied by trainees, and 60 in patients biopsied by faculty doctors. In the overall sample, $56.3 \%$ of patients were men. The distribution of the location of lesions was as follows: stomach, 90.6\%; esophagus, 5.0\%; and duodenum, 4.4\%. Among the gastric lesions, the antrum was the most frequently biopsied area (46.3\%), followed by the body and fundus (33.1\%), angle (8.5\%), and cardia (2.7\%). The baseline characteristics of biopsied lesions were not different between trainees and faculty doctors.

\section{Endoscopic diagnosis vs. pathologic diagnosis}

Among the total lesions, erosion was the most common endoscopic diagnosis $(n=388,32.1 \%$ ), followed by ulcer $(\mathrm{n}=245,20.3 \%)$, polyp ( $\mathrm{n}=147,12.2 \%)$, metaplasia ( $\mathrm{n}=80$, $6.6 \%)$, color change in the mucosa $(n=72,6 \%)$, elevation or nodularity of the mucosa $(n=70,5.8 \%)$, early gastric cancer $(n=38,3.1 \%)$, adenoma $(n=26,2.2 \%)$, advanced gastric cancer $(n=24,2 \%)$, and mucosal irregularity $(n=23$, $1.9 \%)$.

According to the final pathology reports, erosion was the most common histologic diagnosis $(n=413,34.2 \%)$, followed by metaplasia ( $\mathrm{n}=228,18.9 \%$ ), inflammation ( $n=164,13.6 \%$ ), ulcer $(n=110,9.1 \%)$, chronic gastritis $(n=69,5.7 \%)$, adenocarcinoma $(n=49,4.1 \%)$, inflammatory polyp ( $\mathrm{n}=29,2.4 \%$ ), fundic gland polyp ( $\mathrm{n}=26,2.2 \%$ ), hyperplastic polyp ( $n=21,1.7 \%$ ), low grade dysplasia $(n=21$, $1.7 \%)$, congestion ( $n=15,1.2 \%)$, Brunner's gland $(n=8$, $0.7 \%)$, acanthosis $(n=7,0.6 \%)$, papilloma $(n=6,0.5 \%)$, high grade dysplasia $(\mathrm{n}=4,0.3 \%$ ), squamous cell carcinoma $(n=3,0.2 \%)$, lymphangietasia $(n=3,0.2 \%)$, xanthoma $(n=2$, $0.2 \%$ ), carcinoid ( $n=1,0.1 \%)$, and Barrett's esophagus $(n=1$, $0.1 \%)$.

Seven discordant diagnoses were observed in the biopsy-proven adenocarcinoma specimens. The endoscopic diagnoses of these lesions were as follows: ulcer (5), erosion (1), and polyp (1).

\section{Neoplasm detection rates}

After final diagnosis, 76 (6.3\%) neoplastic lesions were detected. The detection rate was lower in trainees than faculty doctors ( $\mathrm{n}=25$ VS. 51, 4.6\% VS. 7.7\%; OR, 0.57; 95\% CI, 0.35 0.93; $P=0.024$ ) (Table 2). The detailed distribution of the neoplasm is as follows; adenocarcinoma $(n=43)$, low grade dysplasia $(n=19)$, signet ring cell carcinoma $(n=6)$, high grade dysplasia $(n=4)$, squamous cell

Table 1. Clinical Characteristics of Biopsied Lesions

\begin{tabular}{lccc}
\hline Characteristic & $\begin{array}{c}\text { Total } \\
(\mathrm{n}=1,208)\end{array}$ & $\begin{array}{c}\text { Lesions biopsied by trainees } \\
(\mathrm{n}=549,45.4 \%)\end{array}$ & $\begin{array}{c}\text { Lesions biopsied by faculty doctors } \\
(\mathrm{n}=659,54.6 \%)\end{array}$ \\
\hline Age $(\mathrm{yr})$ & $60(49 \sim 71)$ & $59(49 \sim 71)$ & $60(49 \sim 71)$ \\
Sex & $680(56.3)$ & $324(59.0)$ & $356(54.0)$ \\
Men & $528(43.7)$ & $225(41.0)$ & $303(46.0)$ \\
Women & & & 5.09 \\
Location & $1,095(90.6)$ & $508(92.5)$ & 0.13 \\
Stomach & $559(46.3)$ & $260(47.4)$ & $299(45.4)$ \\
Antrum & $103(8.5)$ & $45(8.2)$ & $58(8.8)$ \\
Angle & $400(33.1)$ & $191(34.8)$ & $209(31.7)$ \\
Body, fundus & $33(2.7)$ & $12(2.2)$ & $21(3.2)$ \\
Cardia & $53(4.4)$ & $19(3.5)$ & $34(5.2)$ \\
Duodenum & $60(5.0)$ & $22(4.0)$ & $38(5.8)$ \\
Esophagus & & & \\
\hline
\end{tabular}

Values are presented as median (interquartile range) or $\mathrm{n}(\%)$. 
Table 2. Number of Biopsy Samples for Benign vs. Neoplastic Lesions

\begin{tabular}{lccc}
\hline \multicolumn{1}{c}{ Characteristic } & $\begin{array}{c}\text { Total } \\
(\mathrm{n}=1,208)\end{array}$ & $\begin{array}{c}\text { Benign lesions } \\
(\mathrm{n}=1,132,93.7 \%)\end{array}$ & $\begin{array}{c}\text { Neoplastic lesions } \\
(\mathrm{n}=76,6.3 \%)\end{array}$ \\
\hline $\begin{array}{l}\text { Number of biopsy } \\
\text { Endoscopic experience }\end{array}$ & $1(1 \sim 2)$ & $1(1 \sim 2)$ & $1.5(1 \sim 3)$ \\
Faculty members & $659(54.6)$ & $608(53.7)$ & $51(67.1)$ \\
Doctors on fellowship & $549(45.4)$ & $524(46.3)$ & $25(32.9)$ \\
\hline
\end{tabular}

Values are presented as median (interquartile range) or $\mathrm{n}(\%)$.

Table 3. Number of Biopsy Samples Taken by Faculty Doctors vs. Trainees

\begin{tabular}{lccc}
\hline \multicolumn{1}{c}{ Characteristic } & Faculty doctors & Trainees & $P$ value \\
\hline Total lesions & $1(1 \sim 2)$ & $1(1 \sim 2)$ & 0.25 \\
Benign lesions & $1(1 \sim 2)$ & $1(1 \sim 2)$ & 0.54 \\
Neoplastic lesions & $2(1 \sim 3)$ & $1(1 \sim 3)$ & 0.48 \\
\hline
\end{tabular}

Values are presented as median (interquartile range).

Table 4. Distribution of the Number of Biopsy Samples during the Training Period

\begin{tabular}{lcccc}
\hline \multicolumn{1}{c}{ Characteristic } & $\begin{array}{c}\text { May } \\
(\mathrm{n}=473)\end{array}$ & $\begin{array}{c}\text { June } \\
(\mathrm{n}=331)\end{array}$ & $\begin{array}{c}\text { July } \\
(\mathrm{n}=404)\end{array}$ & $P$ value \\
\hline All doctors & $1(1 \sim 2)$ & $1(1 \sim 2)$ & $1(1 \sim 2)$ & 0.98 \\
Trainees (fellow doctors) $)$ & $1(1 \sim 2)$ & $1(1 \sim 2)$ & $1(1 \sim 2)$ & 0.78 \\
Faculty doctors & $1(1 \sim 2)$ & $1(1 \sim 2)$ & $1(1 \sim 2)$ & 0.76 \\
\hline
\end{tabular}

Values are presented as median (interquartile range).

carcinoma $(n=3)$, and carcinoid $(n=1)$.

\section{Number of biopsy}

The median number of biopsy samples was 1 overall (IQR, 1 2), 1.5 (IQR, 1 3) for neoplastic lesions, and 1 (IQR, 1 2) for benign lesions (Table 2). The overall number of samples did not differ between trainees and faculty doctors ( 1 V. $1 ; P=0.25$ ). Faculty doctors took more samples from neoplastic lesions (2 VS. 1), but this difference was not significant ( $P=0.48$ ) (Table 3 ). The number of biopsy samples taken by trainees was not changed over the training time ( $P=0.78)$ (Table 4).

\section{Concordance rates between endoscopic and histologic diagnosis}

The rate of concordance between endoscopic and histologic diagnoses was as follows in the trainees: 54.3\% (total lesions), 52.9\% (benign lesions), and 84\% (neoplastic
Table 5. Concordance Rate between Endoscopic and Histologic Diagnosis

\begin{tabular}{lr}
\hline Characteristic & \multicolumn{1}{c}{ Trainees } \\
\hline Total lesions & $298 / 549(54.3)$ \\
Benign lesions & $277 / 524(52.9)$ \\
Neoplastic lesions & $21 / 25(84.0)$ \\
\hline
\end{tabular}

Values are presented as number/total number (\%).

lesions) (Table 5). The concordance rate-both overall and for lesions determined to be benign-increased over time. The concordance for total lesions in May, June, and July was $43.4 \%, 58.3 \%$, and $60.2 \%$, respectively ( $P=0.002)$. The concordance for benign lesions during those months was $42.0 \%, 57.5 \%$, and $58.3 \%$, respectively ( $P=0.002$ ). The diagnostic method concordance among trainees for neoplastic lesions did not change significantly over the study period: $75 \%$ in May, $80 \%$ in June, and $91.7 \%$ in July $(P=0.51)$ (Table 6).

\section{Multivariable analysis for the detection of neoplastic lesions during endoscopy}

In the multivariable analysis, number of biopsy (OR, 1.87; 95\% CI, 1.52 2.29; $P<0.001$ ), male patients (VS. female; OR, 0.59; 95\% CI, 0.35 0.99; $P=0.04$, and patient's age (OR, 1.04; 95\% CI, 1.02 1.06; $P<0.001)$ were associated with the detection of neoplastic lesions. However, endoscopic experience (trainees VS. faculty doctors) showed no significant association (OR, 0.63; 95\% CI, 0.38 1.06; $P=0.08$ ) (Table 7).

\section{DISCUSSION}

This study investigated the current practice pattern and effects of training in upper endoscopic biopsy according 
Table 6. Distribution of Concordance Rate for the Training Period

\begin{tabular}{lccrc}
\hline \multicolumn{1}{c}{ Characteristic } & May $(\mathrm{n}=473)$ & June $(\mathrm{n}=331)$ & July $(\mathrm{n}=404)$ & $P$ value \\
\hline Total lesions (trainees) & $79 / 182(43.4)^{*}$ & $88 / 151(58.3)^{*}$ & $130 / 216(60.2)$ & 0.002 \\
Neoplastic lesions (trainees) & $6 / 8(75.0)$ & $4 / 5(80.0)$ & $11 / 12(91.7)$ & 0.51 \\
Benign lesions (trainees) & $73 / 174(41.9)^{* *}$ & $84 / 146(57.5)^{* * *}$ & $119 / 204(58.3)$ & 0.002 \\
\hline
\end{tabular}

Values are presented as number/total number (\%).

$* P=0.006, * * P=0.005$.

Table 7. Multivariable Analysis for the Detection of Neoplastic Lesions

\begin{tabular}{lccc}
\hline \multicolumn{1}{c}{ Variable } & OR & $95 \%$ CI & $P$ value \\
\hline Trainees vs. faculty doctors & 0.63 & $0.38 \sim 1.06$ & 0.08 \\
Number of biopsy & 1.87 & $1.52 \sim 2.29$ & $<0.001$ \\
Men vs. women (patients) & 0.59 & $0.35 \sim 0.99$ & 0.04 \\
Patients' age & 1.04 & $1.02 \sim 1.06$ & $<0.001$ \\
\hline
\end{tabular}

to number of biopsy samples and diagnostic outcomes. The optimal number of biopsy samples for gastrointestinal lesions has not been determined. ${ }^{6}$ The data on this topic are limited.

It is obvious that more biopsy samples could improve the diagnostic rate. In the case of ambiguous lesions, when it is difficult to distinguish between neoplastic and non-neoplastic disease, sufficient tissue can be obtained and diagnosis can be determined. ${ }^{7}$ Multivariable analysis in this study also revealed the association of biopsy number and neoplasm detection rate. However, precise endoscopic diagnosis would decrease unnecessary biopsies and would increase necessary biopsy numbers. Therefore, counting biopsy numbers could be meaningless. However, this has not been evaluated in the clinical practice. Precise prediction of the histologic diagnosis during the endoscopic examination is also difficult. Thus, this study evaluated the concordance rate by the standards of neoplasm or not.

In the present study, the number of biopsy samples was associated with the detection of neoplastic lesions (OR, 1.87; 95\% CI, 1.52 2.29; $P<0.001$ ). However, endoscopic biopsy is an invasive procedure that can result in mucosal damage and hemorrhage. Although significant hemorrhage is reported to be rare during diagnostic $\mathrm{EGD},{ }^{8}$ it is difficult to justify a strategy in which the goal is to obtain as much tissue as possible. Moreover, re- peated biopsies or multiple biopsies over wide area can lead to submucosal fibrosis, which impedes therapeutic procedures such as EMR or ESD. In this study, the median number of biopsy samples for neoplastic lesions was 1.5 (IQR, 1 3), which is higher than the number for benign lesions (1; IQR, 1 2; $P<0.001$ ). Considering that the use of a single biopsy specimen carries the risk of targeting the wrong tissue and collecting an insufficient amount of tissue obtainment, these numbers cannot be considered adequate, especially among inexperienced trainees.

This study found no significant difference in biopsy number between faculty doctors and trainees, nor did it find a training effect with respect to the number of samples. Finding a balance between underdiagnosis due to an insufficient number of specimens and complications such as hemorrhage or submucosal fibrosis due to repeated biopsy attempts is important and should be emphasized in training. Developing skills in targeted biopsy should be another objective of training.

In terms of the neoplastic lesion detection rate, there was a difference between trainees and faculty doctors (4.6\% vs. 7.7\%; OR, 0.57; $P=0.024$ ). Additionally, a higher number of biopsy samples were taken from neoplastic lesions by faculty doctors compared with trainees (2 vs. 1), although this difference was not statistically significant $(P=0.48)$. This difference may lead to underdiagnosis of neoplastic lesions among trainees, resulting in lower detection rates and characterization of lesions during the examination. ${ }^{9}$ This potential problem can also be addressed during proper training. The concordance rate between endoscopic and histologic diagnosis improved over time for total and benign lesions but not for the neoplastic lesions. For diagnosis, the characteristics of the lesions (i.e., abnormal mucosal structures, surface pit pattern, color change, and change of microvascular struc- 
tures) were more important to trainees than the absolute number of biopsy samples.

In the analysis of biopsy-proven adenocarcinoma lesions, 7 discordant diagnoses were detected (endoscopic diagnosis: 5 ulcers, 1 polyp, and 1 erosion). Ulcerative lesions and polyps are usually biopsied for histologic confirmation, even when characteristics of pathologic diseases are not present. However, erosive gastritis is common endoscopic diagnosis. According to the analysis of endoscopic diagnosis of biopsied lesions, erosion was the most common endoscopic diagnosis ( $\mathrm{n}=388,32.1 \%)$. Among lesions diagnosed as erosion, 2 low grade dysplasia lesions and 1 adenocarcinoma were detected histologically. Therefore, biopsy of eroded tissue that has characteristics of a neoplastic lesion should be performed.

Metaplasia was the most frequently discordant diagnosis between endoscopy and histology (80 endoscopic metaplasias vs. 228 histologically confirmed metaplasias). This is not surprising, given that there is no standard set of characteristics that can be used to distinguish metaplasia from normal mucosa. ${ }^{10}$ In this study, 53 erosions, 28 ulcers, 9 early gastric cancers, 9 polyps, 6 adenomas, and 3 subepithelial tumors that were endoscopically evaluated were ultimately determined to be metaplasias. Previous studies have also reported low sensitivities as low as $24 \%$ to $24.2 \%$ in the detection of metaplasia by white light endoscopy. ${ }^{11}$ However, other studies have found that light blue crest on NBI was a characteristic of metaplasia ${ }^{12}$ and that confocal endomicroscopy has substantial sensitivity and specificity for detecting metaplasia. ${ }^{13}$ Careful inspection and examination of tissues using these modalities should improve the diagnostic yield.

This study has several limitations. The analyses did not include data on the size of the lesions. Although biopsy forceps were used to measure endoscopic lesions, the inaccuracy of this method could cause bias in the statistical analysis; thus, size was not included in the analyses. Widely adopted optical techniques, such as NBI, were not used consistently or analyzed in terms of training because of the relatively short training period for the fellow doctors ( $<4$ months) to master this skill. Another limitation was the small number of biopsied specimens and the in- clusion of only a single study center. However, this study described the current practice patterns and the effects of training in upper endoscopic biopsy and suggested important education objectives for trainees.

In conclusion, inexperienced endoscopists must pay greater attention to the detection of lesions than to the number of biopsy specimens to avoid overlooking neoplastic diseases. In the lesions suspected as neoplasm, more than 1 biopsy specimen seems to be proper than only a single biopsy specimen.

\section{REFERENCES}

1. Nakanishi Y, Ochiai A, Shimoda T, et al. Heterotopic sebaceous glands in the esophagus: histopathological and immunohistochemical study of a resected esophagus. Pathol Int 1999;49: 364-368.

2. Chiu KW, Chiou SS. Endoscopic biopsy as quality assurance for endoscopic services. PLoS One 2013;8:e78557.

3. Muguruma N, Miyamoto H, Okahisa T, Takayama T. Endoscopic molecular imaging: status and future perspective. Clin Endosc 2013;46:603-610.

4. Cotton PB, Barkun A, Ginsberg G, et al. Diagnostic endoscopy: 2020 vision. Gastrointest Endosc 2006;64:395-398.

5. Templeton A, Hwang JH. Confocal microscopy in the esophagus and stomach. Clin Endosc 2013;46:445-449.

6. Cohen J, Safdi MA, Deal SE, et al; ASGE/ACG Taskforce on Quality in Endoscopy. Quality indicators for esophagogastroduodenoscopy. Am J Gastroenterol 2006;101:886-891.

7. Lee HJ, Kim JI. The biopsy of upper gastrointestinal endoscopy. Korean J Helicobacter Up Gastrointest Res 2012;12:166-170.

8. ASGE Standards of Practice Committee, Anderson MA, Ben-Menachem T, et al. Management of antithrombotic agents for endoscopic procedures. Gastrointest Endosc 2009;70: 1060-1070.

9. Yao K. The endoscopic diagnosis of early gastric cancer. Ann Gastroenterol 2013;26:11-22.

10. Bang BW, Kim HG. Endoscopic classification of intestinal metaplasia. Korean J Helicobacter Up Gastrointest Res 2013; 13:78-83.

11. Lim JH, Kim N, Lee HS, et al. Correlation between endoscopic and histological diagnoses of gastric intestinal metaplasia. Gut Liver 2013;7:41-50.

12. Uedo $\mathrm{N}$, Ishihara $\mathrm{R}$, Iishi $\mathrm{H}$, et al. A new method of diagnosing gastric intestinal metaplasia: narrow-band imaging with magnifying endoscopy. Endoscopy 2006;38:819-824.

13. Guo YT, Li YQ, Yu T, et al. Diagnosis of gastric intestinal metaplasia with confocal laser endomicroscopy in vivo: a prospective study. Endoscopy 2008;40:547-553. 